\title{
Book Reviews by F Calogero
}

University of Rome "La Sapienza", Italy

Three books are reviewed, namely

Masao Nagasawa: Schroedinger Equations and Diffusion Theory. Birkhaeuser, Basel Boston Berlin, 1993. 332 pages.

David Wick (with a mathematical appendix by William Farris): The Infamous Boundary - Seven Decades of Controversy in Quantum Physics. Birkhaeuser. Boston Basel Berlin, 1995. 256 pages.

Luis de la Peña and Ana Maria Cetto: The Quantum Dice - An Introduction to Stochastic Electrodynamics. Kluwer, Dordrecht Boston London, 1996. 515 pages.

These three books treat, from different points of view, a topic I have become recently highly interested into: alternative formulations of quantum mechanics, different from the standard, axiomatic, "Copenhagen" version that constitutes today the conventional wisdom for most physicists.

The first of these books I found the least enlightening: no doubt my fault, for clearly the author - himself a leading scholar on stochastic processes and diffusion theories - assumes more expertise by his readers on such topics than I muster. Indeed it is emphasized that the treatment is rather mathematical than physical:

"The text of the monograph is deductive, partly because the theme is motivated by problems in physics and biology. However, contents of the monograph are purely mathematical, although they might provide new aspects and implications to some problems in applications.

For mathematicians who like an inductive (axiomatic) presentation of mathematical contents, I would recommend reading the text backwards from Chapter 9 to Chapter 3 (once more) as a theory of systems of interacting diffusion processes." (p.12).

(Such intriguing statements abound; another example is the "Remark" on p. 21: "For Brownian motions there are many excellent texbooks and monographs which have different characters. One can refer to e.g. Revuz-Yor (1991) and the references given there." This remark is actually made more puzzling by the lack of Rev-Yor (1991) - a much quoted text - in the final list of References, which is however quite extensive: 16 pages).

Another example of the high standards required by the readers is the habit of quoting paragraphs from classical papers in their original language, be it German (f.i. at page 100) or French (p. 221 - in both cases the original author is E. Schroedinger, who is in fact the main hero of the book); there are however - fortunately for me! - no quotations in Japanese, although Japanese contributions are certainly not underplayed throughout the book.

In any case the main point made in the book is the existence of a correspondence between diffusion-type equations and Schroedinger-type equations: when two functions 
$\phi=\exp (R+S), \hat{\phi}=\exp (R-S)$ satisfy two "dual" diffusion equations, the (wave) function $\psi=\exp (R+i S)$ satisfies a Schroedinger-type equation (and of course the complexconjugate $\bar{\psi}$ of $\psi$ satisfies the complex-conjugate of the Schroedinger equation, while obviously $\phi \hat{\phi}=\psi \bar{\psi}$ ). Much is made of this correspondence, and it is claimed that it provides the answer to the question (in the author's opinion, hitherto unanswered) "What is the Schroedinger equation?". Several applications are moreover reported, including to such variegated topics as "Segregation of a Monkey Population", "Septation of Esterichia Coli" and "The Mass Spectrum of Mesons" (see Section 9.1, 9.3 and 9.4).

The second book I found most enlightening, and delicious reading. Its scope and the level of expertise required of the reader are best explained by reporting verbatim its Table of contents and by quoting from its Introduction:

"Introduction; Prologue I: Atoms; Prologue II; Quanta; Revolution, Part I: Heisenberg's Matrices; Revolution, Part II: Schroedinger's Waves; Uncertainty; Complementarity; The Debate Begins; The Impossibility Theorem; EPR; The Post-War Heresies; Bell's Theorem; Dice Games and Conspiracies; Testing Bell; Loopholes; The Impossible Observed; Paradoxes; Philosphies; Principles; Opinions; Speculations; Postscript; Appendix, by William Farris; Bibliography."

"Of the great scientific controversies of this century, the battle over quantum mechanics involved the widest span of scientific issues, as well as the subtlest arguments. I will try to smooth out the rough parts where I can, but, unavoidably, some topics will tax the reader's intuition, just as they did the author's (not to mention the thousands of investigators who preceded us). As to technical matters, equations other than innocent proportionalities will be eschewed in favor of prose summaries, except occasionally in the Notes. (Footnote 12, see below) However, professor William Farris of the University of Arizona, an expert on the mathematics of quantum mechanics, has written a brief introduction which requires no matrix algebra or even calculus to understand. It is included as an Appendix and will be referred to occasionally in the text; I recommend it highly to anyone who does not experience palpations at the sight of a solitary Greek letter."

(The Footnote reads: "I make one exception in $<<$ Revolution, Part II: Schroedinger's Waves $>>$ for an equation whose bizarre typography, like the arrangement of the saints in medieval paintings, embodies part of the mystery." But I found no such item in the relevant chapter: unless it is the Schroedinger equation itself, which however appears only in the Notes, and never reminded me of "the arrangements of saints in medieval pantings" - my lack of fantasy?).

The book - whose author characterizes himself as "not a professional philosopher but a practicing scientist" - has a historical perspective, but its main focus are the paradoxical (or rather, counterintuitive) aspects of quantum mechanics, in particular the difficulty of reconciling it with a realistic view of the physical world that excludes mysterious actions at a distance (the so-called nonlocal aspects of quantum mechanics). Given the attention to historical developments (including many amusing anedoctes), there are of course many heroes: Schroedinger, Eistein, Bohr and so on (unfortunately the book has no index of subjects and authors); but undoubtedly - and perhaps deservedly - the main one is John Stewart Bell, the Irish scientist born in Belfast in 1928 who passed away suddenly in October 1990, best known for his recognition of the possibility to test experimentally the most puzzling predictions of quantum theory, via what are now universally referred to as 
"Bell's inequalities."

The author ends the book on a skeptical vein, and by outlining what, in his opinion, still needs to be done:

"Quantum mechanics was one of the most successful creations of the human intellect. From the color of neon lights to the hardness of diamonds to the magnetism of electrons, it correctly described a host of physical phenomena. When it worked, it worked. But quantum mechanics was not the end of physics. I had a great time writing this book, and I hope the reader - whatever his or her philosophy - enjoyed reading it. But I am of course dissatisfied. In the last two chapters I advanced many speculations, some no doubt woolly. I have tried to implement some of these speculations mathematically, with indifferent success.

It would give me the greatest pleasure if a reader were to take on these vague speculations about Bell's $<<$ precise theory $>>$ and get it to work. No doubt my concepts will prove inadequate, so by all means invent new ones; they need be neither $<<$ quantum $>>$ nor $<<$ classical $>>$. State your principles, then use the right mathematics, no matter how complex or abstruse; if you are right, others will be only too happy to learn your methodology. Remember that you will have first to explain why quantum mechanics worked so well - just as Einstein had to explain, in 1915, why Newton's theory was so successful. Be sure to make at least one prediction for a plausible experiment, preferably one undreamt of in the old philosophy.

You will have a lot of trouble getting published, and even if you do you will encounter extreme skepticism. So check every detail again and again, and if your theory does not work, discard it. Remember, as Richard Feymann once remarked, the easiest person to fool is yourself.

Good luck."

The third book is an encyclopedic treatise. Here the main heroes are the authors themselves; indeed the book is largely based on previous researches by themselves, but it also contains an exhaustive treatment of the various alternative theories put forward by many different authors to replace or reinterpret the standard version of quantum mechanics: the Bibliography covers 37 pages, the Index of topics and names 25 pages!

The main idea on which this book focuses is the existence of a zero-point fluctuation of the electromagnetic field, a phenomenon which is of course a well-known consequence of quantum theory, and indeed an experimentally well-demonstrated fact. The point of view of the authors, and the scope of the book, are best outlined by quoting from its Preface:

"The consistent introduction of a vacuum (or zero-point) radiation field represents a major step outside the classical framework, although it does not contravene the spirit or the foundations of classical physics. The postulate of its existence could therefore provide a possible clue - or the clue? - for a new understanding of microphysics, to which the qualifications $<<$ classical $>>$ and $<<$ non-classical $>>$, if used in a broad sense, would equally apply. What sort of physics can it be? How much of it depends on the properties of the vacuum field, and how well can they be determined? How important is this electromagnetic vacuum with respect to other vacua?

These are just some of the questions addressed by stochastic electrodynamics. The zero point electromagnetic radiation field, which is the central element of the theory, is considered to be a purely random field (because its average is zero); the intensity (or average energy) of its fluctuations is measured in terms of Planck's constant, which appear thus 
as a new fundamental constant, representing the departure from classical physics. The new theory is therefore expected to differ essentially from the classical, field-free limit, by allowing for the occurrence of fluctuating phenomena characterized by this new constant.

These are just some of the questions addressed by stochastic electrodynamics. The zeropoint electromagnetic radiation field, which is the central element of the theory, is considered to be a purely random field (because its average is zero); the intensity (or average energy) of its fluctuations is measured in terms of Planck's constant, which appears thus as a new fundamental constant, representing the departure from classical physics. The new theory is therefore expected to differ essentially from the classical, field-free limit, by allowing for the occurrence of fluctuating phenomena characterized by this new constant....

Even if still under construction, the results achieved so far by stochastic electrodynamics are not meagre; but they are found scattered and barely systematized so that a general review of them seems to be timely. At the same time it is true that the developments are still in such a stage, that to tell the problems that have already found lasting solutions from those still in demand of reflection, is a risky task that can have different outcomes.

So the present account is by necessity a very personal one, even if in writing it an effort has been made to reduce the arbitrariness in the selection of topics to a minimum. This book attempts to present an overview as fair as possible of most of the main developments up to the beginning of 1994, to characterize as clearly as possible the stage reached by the theory at the present moment, and to identify the problems that have been solved and how, as well as the ones that are still wanting for a solution and why. To keep the size of the book within reasonable limits has resulted in some topics being covered very briefly, and others touched upon only incidentally or even overlooked; as a partial remedy, the bibliography at the end includes all the works known to the authors that are directly related to stochastic electrodynamics."

This book - in spite of the abundance of misprints by which it is marred - will be for a long time an indispensable reference text for all those interested in this problematique; it is indeed a book to be frequently consulted, rather than enjoyed by reading it sequentially from the first page to the last - in contrast to the one mentioned immediately above. Unfortunately its price will force most scholars to make such consultations in the library, rather than at home.

Francesco Calogero 